\title{
reVisão de literatura Prevalência de transtornos mentais entre crianças e adolescentes e fatores associados: uma revisão sistemática
}

\author{
Prevalence of mental disorders among children and \\ adolescents and associated factors: a systematic review
}

Daianna Lima Thiengo', Maria Tavares Cavalcante², Giovanni Marcos Lovisi'

\section{RESUMO}

Objetivo: $\bigcirc$ objetivo deste estudo foi realizar uma revisão sistemática para identificar os transtornos mais prevalentes na infância e adolescência e possíveis fatores associados. Métodos: Várias bases eletrônicas de dados foram pesquisadas. Foram considerados critérios de inclusão: estudos epidemiológicos de base populacional; observacionais; com instrumentos validados; publicados em inglês, espanhol ou português; e que obtiveram pontuação acima de 12 pontos conforme critérios metodológicos do Checklist for Measuring Quality. Resultados: Os transtornos mais frequentes encontrados pelos estudos, respectivamente, foram: de-

\section{Palavras-chave}

Transtornos mentais, crianças, adolescentes, fatores de risco, revisão sistemática.

\section{Keywords}

Mental disorders, children, adolescents, risk factors, systematic review. pressão, transtornos de ansiedade, transtorno de déficit de atenção e hiperatividade (TDAH), transtorno por uso de substâncias e transtorno de conduta. Fatores que mais se mostraram associados aos diferentes transtornos foram: fatores biológicos, fatores genéticos e fatores ambientais. Conclusão: $O$ conhecimento desses transtornos e seus potenciais fatores de risco trazem a possibilidade de desenvolvimento de programas de intervenção focados em prevenir ou atenuar os efeitos destes.

\section{ABSTRACT}

Objective: The objective of this study was to conduct a systematic review to identify the most prevalent disorders in childhood and adolescence and associated factors. Methods: Several electronic databases were searched. Inclusion criteria were: population-based epidemiological studies; observational; with validated instruments; published in English, Spanish or Portuguese; and who achieved a score above 12 points as the methodological criteria Checklist for Measuring Quality. Results: The most frequent disorders found by studies, respectively, were: depression, anxiety disorder, attention deficit hyperactivity disorder (ADHD), substance use disorder and conduct disorder. Factors that were associated with different disorders were biological factors, genetic factors and environmental factors. Conclusion: The knowledge of these disorders and their potential risk factors bring the possibility of developing intervention programs focused on prevent or attenuate their effects.

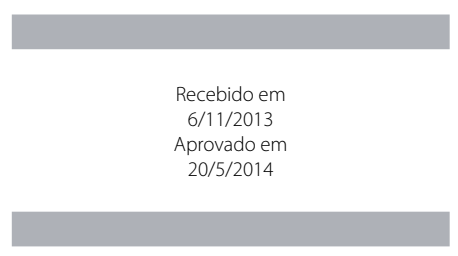

DOI: $10.1590 / 0047-2085000000046$
1 Universidade Federal do Rio de Janeiro (UFRJ), Instituto de Estudos em Saúde Coletiva.

2 UFRJ, Faculdade de Medicina, Instituto de Psiquiatria, Departamento de Psiquiatria.

Endereço para correspondência: Daianna Lima Thiengo

Av. Horacio Macedo, s/n, próximo à Prefeitura Universitária da UFRJ,

Ilha do Fundão, Cidade Universitária, Linha de Saúde Mental e Violências

21941-598 - Rio de Janeiro, RJ, Brasil

Telefone: (21) 99380-8826

E-mail: daianna.thiengo@yahoo.com.br 


\section{INTRODUÇÃO}

Há 13 anos, a Organização Mundial de Saúde (OMS) divulgava um relatório sobre os transtornos mentais e os principais fatores que contribuíam para o surgimento desses transtornos. Esse relatório mencionava os transtornos da infância e adolescência e ressaltava ainda o quanto eles eram comuns e o quanto podiam ser incapacitantes'.

Segundo estimativas da Organização das Nações Unidas $(\mathrm{ONU})$, as crianças e adolescentes representam respectivamente cerca de $30 \%$ e 14,2\% da população mundial ${ }^{2}$. Nessas populações, são encontradas altas taxas de prevalência de transtornos mentais. Em revisão de literatura internacional, a média global da taxa de prevalência de transtornos mentais nessa população foi de 15,8\%. A taxa de prevalência tende a aumentar proporcionalmente com a idade, sendo que a prevalência média entre os pré-escolares foi de 10,2\% e entre os adolescentes, de $16,5 \%{ }^{3}$. No Brasil, estudos registraram taxas de prevalência de 7 a 12,7\% $\%^{4}$. Atualmente, estimativas apontam que uma entre quatro a cinco crianças e adolescentes no mundo apresenta algum transtorno mental ${ }^{5}$.

De acordo com a OMS, existem duas grandes categorias específicas de transtornos mentais na infância e adolescência: transtornos do desenvolvimento psicológico e transtornos de comportamento e emocionais. Os transtornos do desenvolvimento psicológico têm como características o início na primeira ou na segunda infância, com comprometimento ou retardo do desenvolvimento de funções estreitamente ligadas à maturação biológica do sistema nervoso central e a evolução contínua sem remissões nem recaídas. Já os transtornos de comportamentos e emocionais incluem os transtornos hipercinéticos como distúrbios da atividade e da atenção e distúrbios de conduta. Este grupo de transtornos inicia precocemente, durante os primeiros cinco anos de vida, e pode vir acompanhado de um déficit cognitivo e de um atraso específico do desenvolvimento da motricidade e da linguagem?

A literatura científica nos últimos anos tem contribuído para a identificação dos possíveis fatores associados à ocorrência de transtornos mentais nessas populações, os quais podem ser agrupados em: fatores biológicos, relacionados a anormalidades do sistema nervoso central, causadas por lesões, infecções, desnutrição ou exposição a toxinas; fatores genéticos, relacionados à história familiar de transtorno mental; fatores psicossociais, relacionados a disfunções na vida familiar e situações indutoras de estresse; e fatores ambientais, como problemas na comunidade (violência urbana) e tipos de abuso (físico, psicológico e sexual) ${ }^{6,7}$. O conhecimento desses potenciais fatores de risco à saúde mental de crianças e adolescentes traz a possibilidade de desenvolvimento de programas de intervenção focados em prevenir ou atenuar os efeitos desses transtornos ${ }^{1,8}$. E, para sintetizar essas informações, revisões sistemáticas são muito úteis, já que reúnem as informações de um conjunto de estudos que podem apresentar resultados contrários e/ou coincidentes, auxiliando na orientação para investigações futuras.

Portanto, o presente estudo teve como objetivo realizar uma revisão sistemática, reunindo os recentes estudos que avaliaram a prevalência de transtornos mentais na infância e adolescência e possíveis fatores associados.

\section{MÉTODOS}

O método utilizado constituiu-se de uma revisão sistemática da literatura de estudos de base populacional que investigaram a prevalência de transtornos mentais na infância e adolescência e possíveis fatores associados.

As etapas de busca, seleção dos artigos, avaliação da qualidade e extração dos dados foram realizadas de forma independente por dois pesquisadores e as discordâncias entre esses foram resolvidas mediante discussão e consenso.

\section{Estratégia de busca}

Primeiramente, foram selecionados os respectivos descritores para posterior consulta nas bases de dados. As seleções foram realizadas por meio de consulta no Decs - Descritores de Ciências em Saúde. Os descritores selecionados em português, inglês e espanhol, respectivamente, foram: "prevalência", "prevalence", prevalencia"; "transtornos mentais", "mental disorders", "trastornos mentales"; "criança", "child", "niño"; "adolescente", "adolescent", "adolescente"; "fatores de risco", "risk factors", "factores de riesgo".

As bases de dados eletrônicas utilizadas foram: Lilacs/Bireme, SciELO, PubMed/Medline, Scopus e Web of Science, com publicações nos últimos 13 anos, incluindo o período após a divulgação do relatório da OMS ${ }^{1}$ sobre a saúde da infância e adolescência até os dias atuais. A identificação dos descritores citados se concentrou no título ou resumo do artigo.

A partir dos artigos identificados e selecionados na busca eletrônica, também foi realizada a busca manual na seção de referências destes. Os artigos de seção de referência que apresentavam os descritores no título ou resumo também foram selecionados a priori.

\section{Seleção dos estudos}

Na segunda etapa, foi realizada uma seleção dos artigos em que estes deveriam preencher os seguintes critérios de inclusão: estudos epidemiológicos de base populacional, do tipo observacional, longitudinal, seccional e caso-controle; e estudos que utilizaram instrumentos validados para o diagnóstico ou critérios baseados no DSM-IV ou na CID-10.

\section{Avaliação da qualidade dos estudos}

Na terceira etapa, foram aplicados os critérios de exclusão por meio da análise da qualidade dos estudos pelo Checklist 
for Measuring Quality, proposto por Downs \& Black) 9 . É um instrumento aplicável ao delineamento dos artigos para avaliação de sua qualidade, permitindo avaliar a informação, a validade interna (vieses e confundimentos) e externa e a capacidade de detecção de efeito significativo do estudo. Este artigo utilizou a versão composta por 27 itens, sendo excluídas as questões $4,8,13,14,15,19,21,22,23$ e 24 relacionadas a estudos experimentais. Desse modo, ao final, foram avaliados 17 itens, em que os artigos podiam obter até 18 pontos.

Os que apresentaram classificação acima de 70\% (acima de 12 pontos) foram incluídos no estudo por serem considerados pelos autores de maior rigor metodológico. Os mesmos critérios foram utilizados por outros autores em artigos de revisão nacional ${ }^{10,11}$. Nessa fase, o checklist foi aplicado de forma independente por dois pesquisadores e as discordâncias entre eles também foram resolvidas mediante discussão e consenso.

\section{Extração dos dados}

Os estudos que obtiveram maior pontuação foram utilizados na presente revisão e suas informações foram extraídas para comparação. Os dados foram digitados em planilha Excel, que continha as seguintes informações: autores, país e ano de publicação, tipo de estudo, local de realização do estudo, tamanho da amostra, população de interesse, entrevista diagnóstica realizada com ou sem familiar, escore de análise de qualidade dos estudos, diagnóstico, prevalência do transtorno, instrumento utilizado para identificação do transtorno, outras variáveis investigadas pelos estudos e principais resultados. Os estudos foram distribuídos de forma decrescente, segundo ano de publicação.

\section{RESULTADOS}

\section{Resultados da busca e seleção}

A princípio a busca bibliográfica resultou em 7.040 artigos, dos quais 6.986 foram identificados por busca nas bases de dados e 54 foram identificados por busca manual na seção de referências dos artigos encontrados nas bases de dados. A partir da busca dos descritores no título ou resumo, foram identificados apenas 1.043 estudos. Desses, 558 foram excluídos por serem revisões de literatura, como capítulos de livros, e também estudos repetidos nas bases de dados. Dos 485 restantes, 389 foram excluídos por não preencherem os critérios de inclusão (226 foram excluídos por não serem de base populacional e 163 foram excluídos por não serem observacionais). Para a análise final, 96 estudos foram avaliados qualitativamente, sendo 69 excluídos por não alcançarem o escore de qualidade desejado. Com isso, 27 artigos foram analisados na presente revisão (Figura 1).

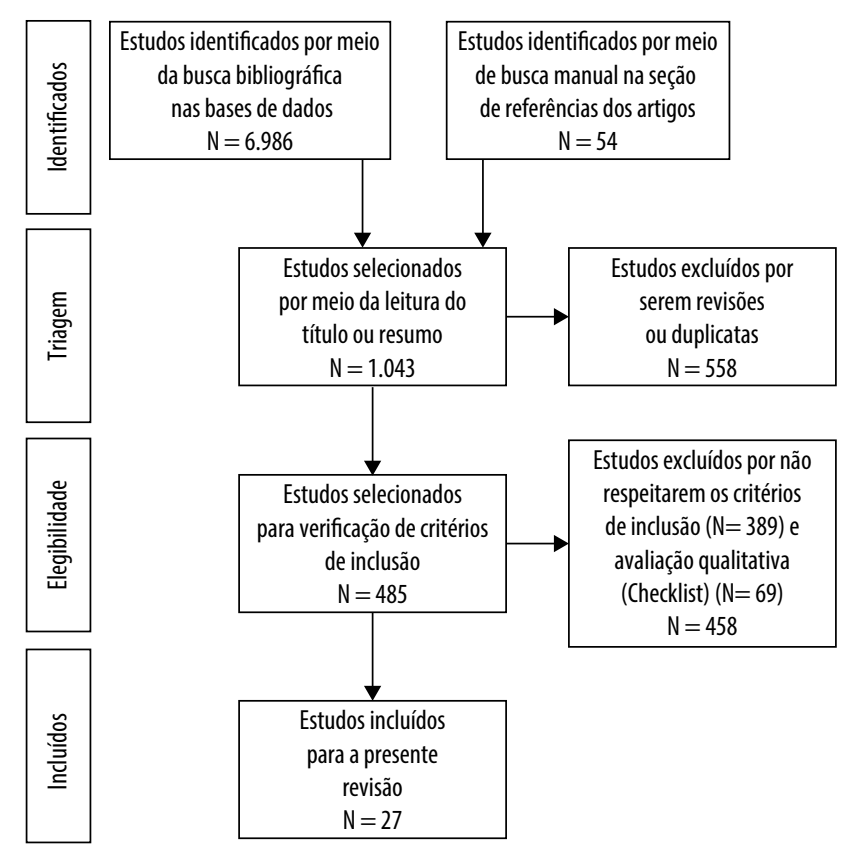

Figura 1. Fluxograma da presente revisão sistemática

\section{Características dos estudos}

A maior parte dos estudos foi realizada em países desenvolvidos ( $n=15)$, sendo a maioria concentrada nos Estados Unidos $(n=10)$. Entre os países em desenvolvimento, dois estudos foram realizados no Brasi $11^{15,28}$.

O desenho de estudo mais frequente também foi o seccional $(n=19)$, seguido pelo desenho de seguimento $(n=6)$. Um estudo foi realizado de forma retrospectiva ${ }^{21}$. A residência foi o principal local de realização dos estudos $(n=20)$, porém um estudo foi realizado com entrevistas nas escolas e, na ausência dos participantes, estes foram também procurados em suas residências ${ }^{23}$.

O número de participantes variou entre 94 e 14.322 . Apenas um estudo utilizou uma amostra menor que 100 participantes ${ }^{31}$. Grande parte dos estudos foi realizada com adolescentes ( $n=12$ ) ou apenas com crianças e também adolescentes ( $n=11$ ). Em sua grande maioria, os estudos entrevistaram as próprias crianças ou adolescentes ( $n=18)$, seis estudos entrevistaram também os familiares e dois estudos identificaram o diagnóstico das crianças apenas com relatos dos familiares ${ }^{13,24}$. Na impossibilidade de resposta pela criança ou pelo adolescente, o estudo de Kinyanda et al. ${ }^{14}$ utilizou também o relato dos familiares.

Em relação à qualidade metodológica dos artigos, a pontuação mínima de 13 pontos foi obtida pela maior parte dos estudos ( $n=16)$ e o valor máximo de 15 pontos, obtidos por dois estudos ${ }^{12,30}$ em que os viéses de seleção foram controlados por meio da randomização. Os estudos que obtiveram menor pontuação não reportaram os valores reais de probabilidade (valor $p$ ou seu nível de significância) ou não relataram os principais fatores confundidores (Tabela 1). 
Tabela 1. Características dos estudos que investigaram a prevalência de transtornos mentais entre crianças e adolescentes nos últimos 13 anos

\begin{tabular}{|c|c|c|c|c|c|c|c|}
\hline Autores & $\begin{array}{l}\text { País/Ano de } \\
\text { publicação }\end{array}$ & Tipo de estudo & Local de estudo & $\begin{array}{l}\text { Tamanho da } \\
\text { amostra }\end{array}$ & População de interesse & $\begin{array}{l}\text { Entrevista diagnóstica } \\
\text { realizada com familiares }\end{array}$ & $\begin{array}{l}\text { Escore de } \\
\text { análise da } \\
\text { qualidade } \\
\text { dos estudos }\end{array}$ \\
\hline Nock et al. ${ }^{12}$ & $\mathrm{EUA} / 2013$ & Seccional & Residência & 6.483 & Adolescentes $\mathrm{M}=\mathrm{NI}$ & Não & 15 \\
\hline Lehaman et al. ${ }^{13}$ & Noruega/2013 & Seccional & Residência & 279 & Crianças $M=8,9$ & Somente familiares & 13 \\
\hline Kinyanda et al..$^{14}$ & Uganda/2013 & Seccional & Residência & 1.587 & Crianças e adolescentes $\mathrm{M}=\mathrm{NI}$ & $\begin{array}{l}\text { No caso de } \\
\text { impossibilidade da } \\
\text { criança, sim }\end{array}$ & 13 \\
\hline Madruga et al..$^{15}$ & Brasil/2013 & Seccional & Residência & 761 & Adolescentes $\mathrm{M}=16,5$ & Não & 13 \\
\hline Lewis et al. ${ }^{16}$ & Reino Unido/2012 & Seguimento & Residência & 337 & Crianças $\mathrm{M}=\mathrm{NI}$ & Também & 14 \\
\hline Benjet et al. ${ }^{17}$ & México/2012 & Seccional & Residência & 3.005 & Adolescentes $M=15,4$ & Não & 13 \\
\hline Vicente et al. ${ }^{18}$ & Chile/2012 & Seccional & Residência & 1.558 & Crianças e adolescentes $\mathrm{M}=\mathrm{NI}$ & Não & 13 \\
\hline Ma et al. ${ }^{19}$ & China/2011 & Seccional & Escola & 3.208 & Adolescentes $M=13,8$ & Não & 14 \\
\hline Siddique et al. ${ }^{20}$ & Índia/2011 & Caso-controle & Residência & $\begin{array}{c}969 \text { Casos } \\
850 \text { Controles }\end{array}$ & Crianças e adolescentes $M=14$ & Não & 14 \\
\hline Peng et al. ${ }^{21}$ & China/2011 & Retrospectivo & Residência & 7.038 & Crianças e adolescentes $\mathrm{M}=\mathrm{NI}$ & Não & 14 \\
\hline Vicente et al. ${ }^{22}$ & Chile/2010 & Seccional & Residência & 254 & Crianças e adolescentes $\mathrm{M}=\mathrm{NI}$ & Não & 14 \\
\hline Merikangas et al..$^{23}$ & $E U A / 2010$ & Seccional & Residência e Escola & 10.123 & Adolescentes $\mathrm{M}=15,2$ & Não & 13 \\
\hline Johnson et al. ${ }^{24}$ & Reino Unido/2010 & Seguimento & Escola & 371 & Crianças $M=10,11$ & Somente familiares & 14 \\
\hline Zinzow et al..$^{25}$ & $E U A / 2009$ & Seccional & Residência & 1.380 & Adolescentes $\mathrm{M}=\mathrm{NI}$ & Não & 13 \\
\hline Allen et al. ${ }^{26}$ & Austrália/2009 & Seguimento & Residência & 1.597 & Adolescentes $\mathrm{M}=\mathrm{NI}$ & Também & 13 \\
\hline Ouyang et al. ${ }^{27}$ & $\mathrm{EUA} / 2008$ & Seguimento & Residência & 14.322 & Crianças $\mathrm{MI}=\mathrm{NI}$ & Também & 14 \\
\hline Cruzeiro et al. ${ }^{28}$ & Brasil/2008 & Seccional & Residência & 1.145 & Adolescentes $\mathrm{M}=\mathrm{NI}$ & Não & 13 \\
\hline Shanahan et al. ${ }^{29}$ & $\mathrm{EUA} / 2008$ & Seccional & Residência & 6.674 & Crianças e adolescentes $\mathrm{M}=\mathrm{NI}$ & Também & 13 \\
\hline Bohon et al..$^{30}$ & $E U A / 2008$ & Seguimento & Escola & 496 & Adolescentes $\mathrm{M}=16,5$ & Não & 15 \\
\hline Piyavhatkul et al. ${ }^{31}$ & Tailândia/2008 & Seccional & Residência & 94 & Crianças e adolescentes $\mathrm{M}=\mathrm{NI}$ & Não & 13 \\
\hline Costello et al. ${ }^{32}$ & $\mathrm{EUA} / 2007$ & Seguimento & $\mathrm{NI}$ & 1.420 & Crianças e adolescentes $\mathrm{Ml}=\mathrm{NI}$ & Não & 13 \\
\hline Roberts et al. ${ }^{33}$ & $\mathrm{EUA} / 2007$ & Seccional & Residência & 4.175 & Crianças e adolescentes $\mathrm{M}=\mathrm{NI}$ & Não & 13 \\
\hline Latimer et al. ${ }^{34}$ & México/2004 & Seccional & Escola & 1.023 & Adolescentes $M=15$ & Não & 13 \\
\hline Canino et al..$^{35}$ & Porto Rico/2004 & Seccional & Residência & 1.886 & Crianças e adolescentes $\mathrm{M}=\mathrm{NI}$ & Também & 13 \\
\hline Glowinski et al. ${ }^{36}$ & $\mathrm{EUA} / 2003$ & Seccional & Residência & 3.416 & Adolescentes $\mathrm{MI}=15,5$ & Não & 13 \\
\hline Martínez-González et al. ${ }^{37}$ & Espanha/2003 & Seccional & Escola & 2.862 & Adolescentes $\mathrm{MI}=\mathrm{NI}$ & Não & 14 \\
\hline Foley et al. ${ }^{38}$ & $\mathrm{EUA} / 2001$ & Seccional & Residência & 850 & Crianças e adolescentes & Também & 14 \\
\hline
\end{tabular}

Nl: não informado; M: média de idade.

\section{Resultados propriamente ditos}

Os transtornos mais frequentes encontrados pelos estudos, respectivamente, foram: depressão, transtornos de ansiedade, transtorno de déficit de atenção e hiperatividade (TDAH), transtorno por uso de substâncias, e transtorno de conduta. Entre esses, os valores de prevalências variaram entre os estudos: depressão - 0,6\% e 30\%; transtornos de ansiedade - 3,3\% e 32,3\%; TDAH - 0,9\% e 19\%, transtorno por uso de substâncias - 1,7\% e 32,1\%; e transtorno de conduta - 1,8\% e $29,2 \%$.

Os instrumentos utilizados foram os mais diversos, sendo os mais utilizados os critérios do Diagnostic and Statistical Manual of Mental Disorders $-4^{\circ}$ Review (DSM-IV), o Child and Adolescent Psychiatric Assessment (CAPA) e o Composite International Diagnóstic Interview (CIDI $)^{39}$, o qual fornece o diagnóstico em concordância com critérios do DSM-IV e da CID-10.

Diversos fatores que poderiam estar relacionados aos transtornos foram investigados pelos estudos. Os fatores mais investigados pelos estudos foram as características sociodemográficas. Fatores genéticos como histórico familiar e fatores ambientais como violência e eventos de vida estressantes também foram muito investigados.

Porém, os fatores que mais se mostraram associados aos diferentes transtornos foram: fatores biológicos, como sexo; fatores genéticos, como histórico familiar de trans- 
torno mental; e fatores ambientais, como presença de violência familiar e comunitária e configuração familiar (morar somente com um dois pais ou nenhum). Fatores psicosso- ciais como baixa autoestima e baixa resiliência foram associados aos transtornos mentais em apenas dois estudos ${ }^{19,30}$ (Tabela 2).

Tabela 2. Características dos estudos que investigaram a prevalência de transtornos mentais entre crianças e adolescentes e principais resultados

\begin{tabular}{|c|c|c|c|c|c|}
\hline Autores & Diagnóstico & Prevalência & $\begin{array}{l}\text { Instrumento Diagnóstico/ } \\
\text { Critério diagnóstico }\end{array}$ & Outras variáveis estudadas & Principais resultados \\
\hline Nock et al..$^{12}$ & $\begin{array}{l}\text { Transtornos de ansiedade } \\
\text { Transtorno de estresse pós-traumático } \\
\text { Depressão } \\
\text { Transtorno bipolar } \\
\text { TDAH } \\
\text { Transtorno opositivo-desafiador } \\
\text { Transtorno de conduta } \\
\text { Transtorno alimentar } \\
\text { Transtorno por uso de álcool e outras substâncias }\end{array}$ & $\begin{array}{c}18,6 \% \\
3,3 \% \\
10,6 \% \\
1,7 \% \\
2,9 \% \\
6,5 \% \\
3,8 \% \\
2,7 \% \\
8,3 \%\end{array}$ & $\begin{array}{l}\text { Composite International } \\
\text { Diagnostic Interview } \\
\text { (CIDI) }\end{array}$ & $\begin{array}{l}\text { Características sociodemográficas, } \\
\text { comorbidades e ideação suicida }\end{array}$ & $\begin{array}{l}\text { A grande maioria dos adolescentes } \\
\text { com histórico de ideação suicida } \\
(89,3 \%) \text { e tentativas }(96,1 \%) \\
\text { preencheu os critérios para pelo } \\
\text { menos uma das } 15 \text { comorbidades }\end{array}$ \\
\hline $\begin{array}{l}\text { Lehaman } \\
\text { et al. }{ }^{13}\end{array}$ & $\begin{array}{l}\text { Transtornos de ansiedade } \\
\text { Transtorno de estresse pós-traumático } \\
\text { Depressão } \\
\text { TDAH } \\
\text { Transtorno opositivo-desafiador } \\
\text { Transtorno de conduta } \\
\text { Transtorno invasivo } \\
\text { Transtorno obsessivo-compulsivo }\end{array}$ & $\begin{array}{c}17,9 \% \\
5 \% \\
1,1 \% \\
19 \% \\
14 \% \\
6,5 \% \\
4,3 \% \\
0,4 \%\end{array}$ & $\begin{array}{l}\text { Developmental and } \\
\text { Well-Being Assessment } \\
\text { (DAWBA) }\end{array}$ & $\begin{array}{l}\text { Características sociodemográficas, } \\
\text { comorbidades e exposição à } \\
\text { violência }\end{array}$ & $\begin{array}{l}\text { Exposição à violência e grave } \\
\text { negligência aumentaram o risco para } \\
\text { transtornos mentais }\end{array}$ \\
\hline $\begin{array}{l}\text { Kinyanda } \\
\text { et al. }{ }^{14}\end{array}$ & Depressão & $8,6 \%$ & $\begin{array}{l}\text { Mini International } \\
\text { Neuropsychiatric } \\
\text { Interview (MINI) }\end{array}$ & $\begin{array}{l}\text { Características sociodemográficas, } \\
\text { violência familiar e comunitária }\end{array}$ & $\begin{array}{l}\text { Crianças e adolescentes expostos } \\
\text { à violência doméstica têm } 94 \% \text { de } \\
\text { chances de ter depressão }(0 \mathrm{R}=1,94 ; \\
\mathrm{P}<0,03) \\
\text { Bairro violento }(0 \mathrm{R}=3,84 ; \mathrm{p}<0,00) \text {, } \\
\text { não morar com os pais }(0 \mathrm{R}=5,39 ; \\
\mathrm{p}<0,00) \text { também se mostraram } \\
\text { associados à depressão }\end{array}$ \\
\hline $\begin{array}{l}\text { Madruga } \\
\text { et al. }{ }^{15}\end{array}$ & Transtorno por uso de substâncias & $14,1 \%$ & $\begin{array}{l}\text { Composite International } \\
\text { Diagnostic Interview } \\
\text { (CIDI) }\end{array}$ & $\begin{array}{l}\text { Características sociodemográficas } \\
\text { e violência doméstica }\end{array}$ & $\begin{array}{l}\text { Sexo masculino }(0 R=3,64 ; p<0,05) \text {, } \\
\text { violência doméstica }(0 R=2,93 ; \\
p<0,05) \text { e maior idade }(0 R=0,71 ; \\
p<0,05) \text { foram associados ao uso de } \\
\text { drogas ilícitas }\end{array}$ \\
\hline $\begin{array}{l}\text { Lewis } \\
\text { et al. }{ }^{16}\end{array}$ & Depressão & $12,5 \%$ & $\begin{array}{l}\text { Child and Adolescent } \\
\text { Psychiatric Assesment } \\
\text { (CAPA) }\end{array}$ & História familiar de depressão & $\begin{array}{l}\text { Pai ou mãe deprimido tem } 2,9 \text { vezes } \\
\text { mais chance de terem filhos menores } \\
\text { de } 12 \text { anos com depressão }(0 R=2,9 ; \\
p=0,00)\end{array}$ \\
\hline $\begin{array}{l}\text { Benjet } \\
\text { et al. }{ }^{17}\end{array}$ & $\begin{array}{l}\text { Transtornos de ansiedade } \\
\text { Depressão } \\
\text { Transtornos por uso de álcool e outras substâncias }\end{array}$ & $\begin{array}{c}32,3 \% \\
10 \% \\
32,1 \%\end{array}$ & $\begin{array}{l}\text { Composite International } \\
\text { Diagnostic Interview } \\
\text { (CIDI) }\end{array}$ & $\begin{array}{l}\text { Características sociodemográficas } \\
\text { e ideação suicida }\end{array}$ & $\begin{array}{l}\text { Adolescentes que trabalham } \\
\text { exclusivamente tem } 1,9 \text { vez mais } \\
\text { chances de apresentarem algum } \\
\text { transtorno mental }(0 R=1,9 ; p<0,00) \\
\text { Aqueles que não trabalham ou } \\
\text { estudam têm } 1,8 \text { vez mais chance de } \\
\text { ideação suicida ( } 0 R=1,8 ; p<0,05)\end{array}$ \\
\hline $\begin{array}{l}\text { Vicente } \\
\text { et al. }{ }^{18}\end{array}$ & $\begin{array}{l}\text { Transtornos de Ansiedade } \\
\text { Depressão } \\
\text { Transtorno alimentar } \\
\text { TDAH } \\
\text { Transtorno opositivo-desafiador } \\
\text { Transtorno de conduta } \\
\text { Transtornos por uso de álcool e outras substâncias }\end{array}$ & $\begin{array}{l}18,5 \% \\
5,9 \% \\
0,3 \% \\
15,1 \% \\
8,7 \% \\
3,7 \% \\
4,8 \%\end{array}$ & $\begin{array}{l}\text { Diagnostic Interview } \\
\text { Schedule for Children } \\
\text { (DISC-IV) }\end{array}$ & $\begin{array}{l}\text { Histórico de transtorno mental na } \\
\text { família e envolvimento familiar }\end{array}$ & $\begin{array}{l}\text { Crianças e adolescentes com histórico } \\
\text { de transtorno mental na família têm } \\
2,4 \text { vezes mais chances de terem } \\
\text { qualquer transtorno mental ( } 0 R= \\
2,4 ; p<0,00) \\
\text { Viver com apenas um dos pais } \\
\text { aumenta em } 2,1 \text { vezes a chance de ter } \\
\text { qualquer transtorno mental ( } 0 \mathrm{R}= \\
2,1 ; \mathrm{p}<0,00)\end{array}$ \\
\hline Ma et al. ${ }^{19}$ & Transtorno do estresse pós-traumático & $2,5 \%$ & $\begin{array}{l}\text { Schedule for Affective } \\
\text { Disorders and } \\
\text { Schizophrenia for } \\
\text { School-Age Children } \\
\text { (KSADS-PL) }\end{array}$ & $\begin{array}{l}\text { Eventos de vida estressante } \\
\text { (catástrofes climáticas) e } \\
\text { resiliência }\end{array}$ & $\begin{array}{l}\text { Quanto menor a resiliência, maior a } \\
\text { probabilidade de ter transtorno do } \\
\text { estresse pós-traumático }(t=-15,09 \text {; } \\
p<0,00)\end{array}$ \\
\hline
\end{tabular}


Tabela 2. Características dos estudos que investigaram a prevalência de transtornos mentais entre crianças e adolescentes e principais resultados

\begin{tabular}{|c|c|c|c|c|c|}
\hline Autores & Diagnóstico & Prevalência & $\begin{array}{l}\text { Instrumento Diagnóstico/ } \\
\text { Critério diagnóstico }\end{array}$ & Outras variáveis estudadas & Principais resultados \\
\hline $\begin{array}{l}\text { Siddique } \\
\text { et al. }\end{array}$ & TDAH $^{\text {a }}$ & $\begin{array}{l}\text { Grupo que } \\
\text { residia } \\
\text { em área } \\
\text { urbana: } \\
11,0 \% \\
\text { Grupo de } \\
\text { área rural: } \\
2,7 \%\end{array}$ & DSM-IVb & Poluição & $\begin{array}{l}\text { Jovens expostos a altos níveis de } \\
\text { poluição têm } 2,06 \text { mais chances de } \\
\text { terem } \operatorname{TDAH}^{\mathrm{a}}(\mathrm{p}<0,00)\end{array}$ \\
\hline Peng et al. ${ }^{21}$ & Transtorno do estresse pós-traumático & $2,05 \%$ & DSM-IVb & $\begin{array}{l}\text { Eventos de vida estressante } \\
\text { (catástrofes climáticas) }\end{array}$ & $\begin{array}{l}\text { Jovens com problemas de } \\
\text { comportamento, diante de um evento } \\
\text { estressante, têm } 5 \text { vezes mais risco de } \\
\text { sofrer com estresse pós-traumático } \\
\left(\mathrm{RR}^{\mathrm{aj}}=5,26 ; \mathrm{p}=0,00\right) \text { e aqueles com } \\
\text { problemas de saúde têm } 3 \text { vezes mais } \\
\text { risco de sofrer com estresse pós- } \\
\text {-traumático }\left(\mathrm{RR}^{\mathrm{aj}}=3,58 ; \mathrm{p}=0,00\right)\end{array}$ \\
\hline $\begin{array}{l}\text { Vicente } \\
\text { et al. }{ }^{22}\end{array}$ & $\begin{array}{l}\text { Transtornos ansiosos, } \\
\text { Transtornos afetivos, } \\
\text { TDAH }^{\mathrm{a}} \\
\text { Transtorno por uso de álcool e outras substâncias } \\
\text { Esquizofrenia }\end{array}$ & $\begin{array}{l}18,9 \% \\
4.1 \% \\
5,0 \% \\
3,6 \% \\
0,9 \%\end{array}$ & $\begin{array}{l}\text { Diagnostic Inteview for } \\
\text { Children (DISC-IV) }\end{array}$ & Características sociodemográficas & $\begin{array}{l}0 \text { sexo feminino esteve menos propenso } \\
\text { a ter transtornos por uso de álcool e } \\
\text { substâncias }(0 R=0,16 ; p<0,01) \\
\text { Ser adolescente esteve menos } \\
\text { propenso a apresentar transtorno do } \\
\text { comportamento }(0 R=0,20 ; p<0,05)\end{array}$ \\
\hline $\begin{array}{l}\text { Merikangas } \\
\text { et al. }{ }^{23}\end{array}$ & $\begin{array}{l}\text { Transtornos de ansiedade } \\
\text { Depressão } \\
\text { Transtorno bipolar } \\
\text { TDAH }^{\text {a }} \\
\text { Transtorno opositivo-desafiador } \\
\text { Transtorno de conduta } \\
\text { Transtorno alimentar } \\
\text { Transtorno por uso de álcool e outras substâncias }\end{array}$ & $\begin{array}{c}31,9 \% \\
11,7 \% \\
2,9 \% \\
8,7 \% \\
12,6 \% \\
6,8 \% \\
2,7 \% \\
11,4 \%\end{array}$ & $\begin{array}{l}\text { Composite International } \\
\text { Diagnostic Interview } \\
\text { (CIDI) }\end{array}$ & Características sociodemográficas & $\begin{array}{l}0 \text { sexo masculino teve maior } \\
\text { probabilidade de ter transtornos de } \\
\text { comportamento }(0 \mathrm{R}=24,8 ; \mathrm{P}<0,00) \\
\text { Adolescentes com familiares com } \\
\text { baixo nível educacional tiveram maior } \\
\text { probabilidade de apresentar qualquer } \\
\text { transtorno mental } \\
(0 \mathrm{R}=19,7 ; \mathrm{p}<0,00)\end{array}$ \\
\hline $\begin{array}{l}\text { Johnson } \\
\text { et al. }{ }^{24}\end{array}$ & $\begin{array}{l}\text { Transtorno de ansiedade } \\
\text { Depressão } \\
\text { TDAH }^{\mathrm{a}} \\
\text { Transtorno opositivo-desafiador } \\
\text { Transtorno de conduta } \\
\text { Transtorno obsessivo-compulsivo } \\
\text { Transtorno do estresse pós-traumático } \\
\text { Autismo }\end{array}$ & $\begin{array}{c}9,1 \% \\
2,2 \% \\
14,4 \% \\
10,3 \% \\
1,8 \% \\
2,4 \% \\
0,5 \% \\
8 \%\end{array}$ & $\begin{array}{l}\text { Development And Well } \\
\text { Being Assessment } \\
\text { (DAWBA) }\end{array}$ & $\begin{array}{l}\text { Prematuridade e baixo peso ao } \\
\text { nascer }\end{array}$ & $\begin{array}{l}\text { Crianças prematuras são } \\
3 \text { vezes mais propensas a apresentar } \\
\text { qualquer transtorno psiquiátrico }(0 R \\
=3,2 ; \mathrm{p}=0,00)\end{array}$ \\
\hline $\begin{array}{l}\text { Zinzow } \\
\text { et al. }\end{array}$ & Depressão; Transtorno de estresse pós-traumático & $\begin{array}{c}10,9 \% \\
7,1 \%\end{array}$ & DSM-IVb & Violência familiar e comunitária & $\begin{array}{l}\text { Crianças e adolescentes que viviam } \\
\text { em comunidades muito violentas } \\
\text { têm } 2,71 \text { vezes mais chance de terem } \\
\text { transtorno do estresse pós- } \\
\text {-traumático ( } p<0,00) \text {. E quando } \\
\text { do sexo masculino, } 2,58 \text { vezes mais } \\
\text { chances ( } p<0,01 \text { ) } \\
\text { Crianças e adolescentes que sofrem } \\
\text { violência familiar têm } 3,07 \text { vezes } \\
\text { mais chance de terem depressão ( } p \\
<0,05 \text { ). E quando do sexo masculino, } \\
3,8 \text { vezes mais chances }(p<0,00)\end{array}$ \\
\hline Allen et al. ${ }^{26}$ & Transtornos alimentares & $6 \%$ & DSM-IV & $\begin{array}{l}\text { Características sociodemográficas, } \\
\text { preditores pré-natais e sintomas } \\
\text { ansiosos e depressivos }\end{array}$ & $\begin{array}{l}\text { Sexo feminino }(p<0,001) \text { e excesso } \\
\text { de peso percebido pelos pais } \\
(p<0,05) \text { foram associados aos } \\
\text { transtornos alimentares }\end{array}$ \\
\hline $\begin{array}{l}\text { Ouyang } \\
\text { et al. } .^{27}\end{array}$ & TDAH & $8,3 \%$ & DSM-IV & $\begin{array}{l}\text { Características sociodemográficas } \\
\text { e violência }\end{array}$ & $\begin{array}{l}\text { Crianças com predominância } \\
\text { desatenta estiveram associadas à } \\
\text { negligência }(0 R=1,6 ; p<0,001) \text {, } \\
\text { abuso físico }(0 R=1,6, p<0,05) \text { e } \\
\text { abuso sexual }(0 R=2,6 ; p<0,001) \\
\text { Já a predominância hiperativa esteve } \\
\text { associada à negligência }(0 R=1,5 ; p< \\
0,05) \text { e abuso físico } \\
(0 R=1,6 ; p<0,05)\end{array}$ \\
\hline
\end{tabular}


Tabela 2. Características dos estudos que investigaram a prevalência de transtornos mentais entre crianças e adolescentes e principais resultados

\begin{tabular}{|c|c|c|c|c|c|}
\hline Autores & Diagnóstico & Prevalência & $\begin{array}{l}\text { Instrumento Diagnóstico/ } \\
\text { Critério diagnóstico }\end{array}$ & Outras variáveis estudadas & Principais resultados \\
\hline $\begin{array}{l}\text { Cruzeiro } \\
\text { et al. }^{28}\end{array}$ & Transtorno de conduta & $29,2 \%$ & $\begin{array}{l}\text { Mini International } \\
\text { Neuropsychiatric } \\
\text { Interview } \\
\text { (MINI KID) }\end{array}$ & $\begin{array}{l}\text { Características sociodemográficas, } \\
\text { uso de substâncias, bullying e } \\
\text { sintomas depressivos }\end{array}$ & $\begin{array}{l}\text { Sexo masculino aumentou em } 2,04 \text { a } \\
\text { chance de ter transtorno de conduta } \\
(p=0,00) \\
\text { Uso de drogas no último mês } \\
\text { aumentou em } 3 \text { vezes a chance de } \\
\text { transtorno de conduta ( } p=0,001) \\
\text { Sofrer bullying aumentou em } 2,02 \\
\text { vezes a chance de ter transtorno de } \\
\text { conduta ( } p=0,001)\end{array}$ \\
\hline $\begin{array}{l}\text { Shanahan } \\
\text { et al. }{ }^{29}\end{array}$ & $\begin{array}{l}\text { Transtorno de ansiedade generalizada } \\
\text { Depressão } \\
\text { TDAH } \\
\text { Transtorno de conduta }\end{array}$ & $\begin{array}{l}1,7 \% \\
2,2 \% \\
0,9 \% \\
2,06 \%\end{array}$ & $\begin{array}{l}\text { Child and Adolescent } \\
\text { Psychiatric Assessment } \\
\text { (CAPA) }\end{array}$ & $\begin{array}{l}\text { Abuso sexual e eventos de vida } \\
\text { estressantes }\end{array}$ & $\begin{array}{l}\text { Crianças e adolescentes que sofreram } \\
\text { abuso sexual têm } 4,91 \text { vezes mais } \\
\text { chance de apresentarem transtorno } \\
\text { de conduta }(p<0,05)\end{array}$ \\
\hline $\begin{array}{l}\text { Bohon } \\
\text { et al. }^{30}\end{array}$ & $\begin{array}{l}\text { Depressão } \\
\text { Transtorno alimentar (bulimia) } \\
\text { Transtorno por uso de substâncias }\end{array}$ & $\begin{array}{l}4,2 \% \\
0,4 \% \\
8,9 \%\end{array}$ & $\begin{array}{l}\text { Schedule for Affective } \\
\text { Disorders and } \\
\text { Schizophrenia for } \\
\text { School-Age Children } \\
\text { (KSADS-PL) }\end{array}$ & $\begin{array}{l}\text { Eventos de vida estressante e } \\
\text { autoestima }\end{array}$ & $\begin{array}{l}\text { Crianças e adolescentes com baixa } \\
\text { autoestima apresentam } 1,45 \text { mais } \\
\text { chances de ter depressão na presença } \\
\text { de eventos estressantes }(p=0,02)\end{array}$ \\
\hline $\begin{array}{l}\text { Piyavhatkul } \\
\text { et al. }{ }^{31}\end{array}$ & $\begin{array}{l}\text { Transtorno de ansiedade } \\
\text { Transtorno do estresse pós-traumático } \\
\text { Depressão } \\
\text { TDAH } \\
\text { Retardo mental }\end{array}$ & $\begin{array}{l}3,3 \% \\
33 \% \\
9,6 \% \\
4,3 \% \\
4,3 \%\end{array}$ & DSM-IVb & $\begin{array}{l}\text { Eventos de vida estressante } \\
\text { (catástrofes da natureza) }\end{array}$ & $\begin{array}{l}\text { Crianças entre } 6 \text { e } 10 \text { anos que } \\
\text { vivenciaram uma catástrofe da } \\
\text { natureza tinham } 7 \text { vezes mais chance } \\
\text { de sofrerem com transtorno do estresse } \\
\text { pós-traumático ou qualquer outro } \\
\text { transtorno mental }(0 R=7,63 ; p=0,00)\end{array}$ \\
\hline $\begin{array}{l}\text { Costello } \\
\text { et al. }^{32}\end{array}$ & Depressão & $30 \%$ & $\begin{array}{l}\text { Child and Adolescent } \\
\text { Psychiatric Assessment } \\
\text { (CAPA) }\end{array}$ & $\begin{array}{l}\text { Características sociodemográficas, } \\
\text { histórico familiar e baixo peso } \\
\text { ao nascer }\end{array}$ & $\begin{array}{l}\text { Baixo peso ao nascer e mãe com } \\
\text { menos de } 18 \text { anos no momento do } \\
\text { parto foram associados à depressão } \\
\text { entre os jovens (respectivamente } 0 R \\
=7,6 ; p<0,01 ; 0 R=3,6 ; p=0,01 \text { ) }\end{array}$ \\
\hline $\begin{array}{l}\text { Latimer } \\
\text { et al. }{ }^{34}\end{array}$ & Transtornos por uso de álcool e outras substâncias & $6,1 \%$ & DSM-IVb & Características sociodemográficas & $\begin{array}{l}\text { Jovens que tinham um irmão que } \\
\text { usou drogas apresentaram duas vezes } \\
\text { mais chances de usar álcool - } \\
(0 \mathrm{R}=2,4 ; \mathrm{p}<0,05) \\
\text { Jovens com amigos que usam drogas } \\
\text { apresentaram quatro vezes mais } \\
\text { chances de usar drogas } \\
(0 \mathrm{R}=4,7 ; \mathrm{p}<0,05)\end{array}$ \\
\hline $\begin{array}{l}\text { Canino } \\
\text { et al. }{ }^{35}\end{array}$ & $\begin{array}{l}\text { Transtornos de ansiedade } \\
\text { Depressão } \\
\text { TDAH }^{\text {a }} \\
\text { Transtorno opositivo-desafiador } \\
\text { Transtorno de conduta } \\
\text { Transtornos por uso de álcool e outras substâncias }\end{array}$ & $\begin{array}{l}6,9 \% \\
3,0 \% \\
8,0 \% \\
2,0 \% \\
5,5 \% \\
1,7 \%\end{array}$ & DSM-IVb & $\begin{array}{l}\text { Características sociodemográficas } \\
\text { e funcionalidade }\end{array}$ & $\begin{array}{l}\text { Meninos tinham duas vezes mais } \\
\text { chances de apresentar TDAH }(0 R=2,0 ; \\
\mathrm{p}<0,05) \text {, enquanto as meninas tinham } \\
\text { cinco vezes mais chance de apresentar } \\
\text { depressão }(0 \mathrm{R}=5,2 ; \mathrm{p}<0,05) \\
\text { Jovens com pais separados tinham até } \\
40 \% \text { de chance de apresentar algum } \\
\text { diagnóstico ( } 0 \mathrm{R}=1,40 ; \mathrm{p}<0,05) \\
\text { Aqueles que moravam nos centros } \\
\text { urbanos aumentavam o risco em até } \\
90 \% \text { de apresentar qualquer transtorno } \\
\text { mental }(0 \mathrm{R}=1,90 ; \mathrm{p}<0,05)\end{array}$ \\
\hline
\end{tabular}


Tabela 2. Características dos estudos que investigaram a prevalência de transtornos mentais entre crianças e adolescentes e principais resultados

\begin{tabular}{|c|c|c|c|c|c|}
\hline Autores & Diagnóstico & Prevalência & $\begin{array}{l}\text { Instrumento Diagnóstico/ } \\
\text { Critério diagnóstico }\end{array}$ & Outras variáveis estudadas & Principais resultados \\
\hline $\begin{array}{l}\text { Glowinski } \\
\text { et al. }^{36}\end{array}$ & Depressão & $8,9 \%$ & DSM-IVb & Histórico familiar & $\begin{array}{l}0 \text { componente genético contribuiu } \\
\text { em } 40 \% \text { para o risco para depressão }\end{array}$ \\
\hline $\begin{array}{l}\text { Martínez- } \\
\text { González } \\
\text { et al. }{ }^{37}\end{array}$ & Transtorno alimentar & $3,1 \%$ & DSM-IVb & Características sociodemográficas & $\begin{array}{l}\text { Um estado civil dos pais diferente } \\
\text { de "estar casado" foi associado com } \\
\text { um risco significativamente maior } \\
\text { para transtorno alimentar ( } 0 \text { R: } 2,0 \text {; } \\
p=0,02 \text { ) }\end{array}$ \\
\hline $\begin{array}{l}\text { Foley } \\
\text { et al. }^{38}\end{array}$ & $\begin{array}{l}\text { Transtorno de ansiedade de separação } \\
\text { Depressão } \\
\text { Transtorno opositivo-desafiador } \\
\text { Transtorno de conduta }\end{array}$ & $\begin{array}{l}4,86 \% \\
0,61 \% \\
1,51 \% \\
2,61 \%\end{array}$ & $\begin{array}{l}\text { Child and Adolescent } \\
\text { Psychiatric Assessment } \\
\text { (CAPA) }\end{array}$ & $\begin{array}{l}\text { Características sociodemográficas } \\
\text { e histórico familiar }\end{array}$ & $\begin{array}{l}\text { Depressão materna aumentou em } \\
2 \text { vezes a chance de transtorno de } \\
\text { conduta nos jovens }(0 R=2,0 ; p= \\
0,03) \\
\text { Alcoolismo materno aumentou em } \\
3 \text { vezes a chance de transtorno de } \\
\text { conduta ( } 0 R=3,14 ; p=0,02) \\
\text { Transtorno do pânico paterno } \\
\text { aumentou em } 2 \text { vezes a chance de } \\
\text { transtorno de ansiedade de separação } \\
\text { nos jovens ( } 0 R=2,37 ; p=0,05)\end{array}$ \\
\hline
\end{tabular}

'Transtorno de Déficit de Atenção e Hiperatividade.

${ }^{b}$ Diagnostic and Statistical Manual of Mental Disorders - DSM 4a Review.

$\mathrm{RR}^{\mathrm{aj}}$. Risco relativo ajustado.

\section{DISCUSSÃO}

\section{Prevalência dos transtornos mentais em crianças e adolescentes}

Os estudos selecionados na presente revisão apresentaram taxas de prevalência dos transtornos mentais compatíveis com outros estudos presentes na literatura científica ao longo dos anos. Em relação aos critérios diagnósticos utilizados pelos estudos, o Diagnostic and Statistical Manual of Mental Disorders (DSM-IV) pareceu vigorar entre os estudos internacionais, principalmente os estudos americanos. Este manual fornece critérios de diagnóstico para a generalidade dos transtornos mentais, incluindo componentes descritivos, de diagnóstico e de tratamento, constituindo um instrumento de trabalho de referência para os profissionais da área. Além de manuais como o DSM-IV, os estudos utilizaram entrevistas diagnósticas, amplamente utilizadas em psiquiatria, como o Schedule for Affective Disorders and Schizophrenia for School-Age Children (KADS-PL) e o Composite International Diagnostic Interview (CIDI), que também se baseiam nos critérios diagnósticos do DSM-IV e da CID-10. Ainda assim, há um contínuo debate científico sobre a validade da construção e a confiabilidade prática das categorias diagnósticas e critérios do DSM. Muitos pesquisadores afirmam que esse sistema de classificação faz distinções categoricamente exageradas entre o normal e o anormal ${ }^{40}$, ocasionando altas taxas de prevalência, porém os estudos presentes nesta revisão, em sua maioria, não apresentaram taxas muito elevadas.

Nos últimos anos, a literatura científica tem chamado a atenção para a ocorrência de transtornos depressivos em crianças e adolescentes. Na presente revisão, mais da me- tade dos estudos encontraram prevalências entre 5,9\% e $12,5 \%$. Estimativas brasileiras apontam que $0,4 \%$ a $3 \%$ das crianças apresentam quadros de depressão ${ }^{41}$. Já em estudos americanos, a prevalência entre crianças e adolescentes varia de $3 \%$ a 5\% $\%^{42}$. Em um estudo alemão, Mehler-Wex e Kolch ${ }^{43}$ identificaram uma prevalência de depressão em crianças e adolescentes de 8,9\%. Essas diferenças podem ser em decorrência de vários fatores como diferentes instrumentos diagnósticos utilizados, local do estudo (em residências ou escolas), população estudada (amostra ou população total). Além disso, o transtorno depressivo pode ser subdiagnosticado dado a sua similaridade com outros transtornos assim como a presença de comorbidades, como TDAH, transtornos de ansiedade, de conduta, agressividade, que podem persistir após cessar o episódio depressivo ${ }^{44}$.

Já os transtornos ansiosos são os quadros psiquiátricos mais comuns tanto em crianças quanto em adultos, com uma prevalência em crianças e adolescentes estimada em torno de $9 \%{ }^{45}$. Na presente revisão, entre os estudos que investigaram a prevalência desse transtorno, mais da metade encontraram prevalências entre 9,1\% e 32,3\%. Em crianças, o desenvolvimento emocional influi sobre as causas e a maneira como se manifestam os medos e as preocupações tanto normais quanto patológicos. Diferentemente dos adultos, crianças podem não reconhecer seus medos como exagerados ou irracionais, especialmente as menores ${ }^{45}$.

A média de prevalência de TDAH nos estudos foi de 8,3\%. Apenas quatro estudos encontraram prevalências acima de 10\%. Do mesmo modo, Golfeto e Barbosa ${ }^{46}$ apresentaram estudos com prevalências que variam de $1 \%$ a $20 \%$. Alguns estudos epidemiológicos de base comunitária, realizados com 
crianças, mostraram que a prevalência de TDAH estava entre $4 \%$ e $12 \%{ }^{47}$. Um estudo brasileiro realizado entre crianças e adolescentes de 6 a 15 anos de escolas públicas encontrou uma prevalência de $17 \%{ }^{48}$.

Essas diferenças nas prevalências dos estudos podem ser explicadas, já que os estudos utilizaram populações com faixa etária distinta. Por exemplo, na infância as características principais do transtorno são a desatenção, a hiperatividade e a impulsividade ${ }^{49}$. Porém, no início da adolescência, o transtorno é relativamente estável, atenuando-se durante o final da adolescência, sendo essa queda mais significativa para sintomas de hiperatividade e impulsividade, consequentemente diminuindo sua gravidade ${ }^{50}$. Outros fatores, como baixo nível socioeconômico e entrevistas realizadas com informantes distintos (pais, educadores ou a própria criança), podem explicar as diferenças nas prevalências encontradas pelos estudos.

Nesta revisão, metade dos estudos encontrou prevalências de transtorno por uso de substâncias entre 8,3\% e 32,1\%. Esses estudos foram realizados somente com adolescentes. Em 1997, um monitoramento realizado no Estados Unidos com 49.500 estudantes encontrou prevalências de 11,8\% entre jovens de 13 anos, 18,2\% entre jovens de 15 anos e $20,7 \%$ entre jovens de 17 anos $^{51}$. Essas prevalências podem ser explicadas já que a dependência por uso de substâncias é o transtorno coexistente mais frequente entre portadores de transtornos mentais na adolescência, como depressão, transtornos de ansiedade e conduta ${ }^{52}$, sendo fundamental o correto diagnóstico das patologias envolvidas.

O uso de substâncias psicoativas ilegais entre adolescentes tem sido um grave problema social e de saúde no Brasilil ${ }^{53}$. Em 2005, o Centro Brasileiro de Informações sobre Drogas (CEBRID) realizou um inquérito entre jovens estudantes de 10 a 19 anos que mostrou uma tendência ao aumento do uso dos inalantes, da maconha, da cocaína e de crack em determinadas capitais, principalmente do sudeste e nordeste ${ }^{54}$. Já o estudo de Madruga et al..$^{15}$ realizado com 761 adolescentes em 143 municípios indicou uma prevalência do transtorno por uso de substâncias de 14,1\%, evidenciando a necessidade de políticas públicas para a questão.

A prevalência de transtorno de conduta entre crianças e adolescentes tem crescido nas últimas décadas, especialmente em áreas urbanas, oscilando de menos de 1\% a mais de $10 \%{ }^{55}$. Na presente revisão, a média de prevalência foi de $4,17 \%$. Em comparação, um estudo canadense encontrou uma prevalência em torno de 5,5\%56. Já em países em desenvolvimento essa prevalência ficou em torno de 30\%28,57. Os transtornos de conduta têm início na infância e adolescência ${ }^{58}$ e merecem atenção, já que a persistência dos comportamentos que caracterizam o transtorno pode conduzir a um diagnóstico de transtorno de personalidade antissocial na idade adulta ${ }^{59}$. As diferenças nas prevalências encontradas nos estudos podem ser decorrentes dos diferentes mé- todos para coleta de dados. Também é esperado que, em amostras clínicas, a prevalência seja maior (familiares buscam tratamento ou são encaminhados) e, em instituições socioeducativas, mais elevada ainda. Em amostras comunitárias, como os estudos presentes nesta revisão, a prevalência de transtornos mentais costuma ser menor.

Mesmo que esta revisão só tenha encontrado três estudos sobre os transtornos globais do desenvolvimento, como autismo e retardo mental, esses transtornos merecem atenção. Em todo o mundo suas prevalências alcançam 1\% da população infantojuvenil60,61. Na presente revisão, os estudos encontraram, entre crianças, taxas em torno de 5,3\%. Desse modo, seu diagnóstico e sua investigação de fatores associados e agravantes são de suma importância para o prognóstico destes. Os transtornos globais do desenvolvimento e o retardo mental estão associados a uma sobrecarga de seus familiares, o que, por sua vez, pode interferir negativamente na adesão ao tratamento e piora do prognóstico ${ }^{62,63}$.

\section{Fatores associados aos transtornos mentais em crianças e adolescentes}

De acordo com a literatura, fatores biológicos, genéticos e ambientais foram associados aos transtornos mentais entre crianças e adolescentes. Na presente revisão, vários fatores foram associados a mais de um transtorno.

Na nossa revisão, encontramos estudos que mostraram uma associação entre sexo masculino e TDAH ${ }^{35}$, transtorno de conduta ${ }^{28,33}$ e também por uso de substâncias ${ }^{15,28}$.

A associação entre o sexo masculino e TDAH tem sido ressaltada por alguns estudos populacionais devido à diferença da proporção entre os sexos masculino e feminino. Provavelmente, essa diferença se deve ao fato de as meninas apresentarem menos sintomas de agressividade/impulsividade e conduta, causando menos incômodo às famílias e à escola, fazendo com que elas sejam menos encaminhadas ao tratamento ${ }^{64}$.

Em relação ao sexo masculino e ao transtorno de conduta, é sabido na literatura que há uma associação maior entre esses, provavelmente por algumas diferenças que caracterizam o sexo masculino e o sexo feminino ${ }^{28}$. Uma dessas diferenças é a expressão da agressividade, maior entre meninos ${ }^{65}$, o que justificaria em parte a maior prevalência do transtorno de conduta.

Já em relação ao sexo masculino e os transtornos por uso de substâncias, o estudo de Moon et al. ${ }^{66}$ verificou que o sexo masculino inicia o uso de drogas com colegas do mesmo sexo, irmãos, primos, ou com estranhos do sexo masculino, geralmente em locais públicos. O estudo de Latimer et al. ${ }^{34}$ também evidenciou tal resultado. Ainda segundo Moon et al.66, os rapazes também iniciam o uso de drogas em idade mais precoce e têm maior risco de receberem ofertas para usarem drogas do que o sexo feminino. Para Moon et al. ${ }^{66}$, na cultura feminina, geralmente o uso de drogas não se inicia 
com parceiros masculinos até que comecem a se relacionar emocionalmente; assim, o sexo feminino teria menos risco de se envolver com drogas em idades mais precoces. O estudo de Vicente et al. ${ }^{22}$ encontrou no sexo feminino um fator protetivo contra a dependência de álcool e drogas. Para o sexo masculino é dada liberdade e independência em idade mais precoce do que para o sexo feminino, o que facilitaria o início do uso de drogas em locais públicos e, consequentemente, aumentaria o risco do início do uso mais precocemente ${ }^{67}$.

Também houve associação entre sexo masculino e transtorno do estresse pós-traumático e depressão ${ }^{25}$. O estudo de Zinzow et al. ${ }^{25}$ encontrou uma associação entre sexo masculino e depressão em ambientes de violência. Segundo o estudo de Costa et al. ${ }^{68}$, adolescentes do sexo masculino estão mais sujeitos a sofrer violência no ambiente comunitário. E, como consequência, crescer em um ambiente de tensão pode contribuir para o desenvolvimento de comportamentos agressivos e/ou defensivos, manifestando-se em retraimento e depressão ${ }^{69}$.

Já em relação ao sexo feminino, os estudos mostraram uma maior associação com transtorno depressivo ${ }^{35}$ e alimen$\operatorname{tar}^{26}$. Segundo Versiani et al. ${ }^{70}$, o sexo masculino apresenta taxas de depressão um pouco maiores do que ao sexo feminino na infância, entretanto na adolescência ocorre uma modificação com um predomínio do sexo feminino sobre o sexo masculino. Alguns estudos envolvendo crianças e adolescentes demonstraram que a diferença de gênero na prevalência de depressão se manifesta primeiramente entre os 11 e 14 anos, assim se mantendo no decorrer da vida adulta, o que pode sugerir um papel determinante dos hormônios sexuais ${ }^{71}$. As mudanças hormonais também têm sido a explicação para a maior prevalência de insônia entre o sexo feminino ${ }^{72}$. Em relação aos transtornos alimentares, diversos estudos comprovam a correlação entre insatisfação com a imagem corporal e a prevalência de sintomas de transtorno alimentar entre o sexo feminino. Em alguns estudos, a insatisfação corporal é a principal justificativa dos transtornos alimentares, estando ainda associada com a depressão $0^{73}$ e a baixa autoestima ${ }^{74}$.

O histórico de transtorno mental na família também se mostrou associado a depressão, transtorno de ansiedade e, no estudo de Vicente et al. ${ }^{18}$, esse fator esteve associado à ocorrência de qualquer transtorno mental. Diversos estudos sugerem um modelo complexo de transmissão genética para os transtornos mentais na infância e que perduram pela adolescência. A manifestação do transtorno depende da presença de um conjunto de genes que interagem entre si resultando em uma fisiopatologia complexa. Outro aspecto inerente aos transtornos mentais na infância e adolescência é a influência do meio sobre a expressão gênica e sobre a modulação da atividade mental ${ }^{75}$.

Em relação aos fatores ambientais, a presença de violência comunitária e familiar esteve associada à ocorrência de diversos transtornos, como depressão ${ }^{14,25}$, transtorno de condu- ta $^{28,29}, \mathrm{TDAH}^{27}$, transtorno por uso de substâncias psicoativas ${ }^{15}$ e transtorno do estresse pós traumático ${ }^{25}$. Flores e Caminha ${ }^{76}$ relatam que uma maneira de explicar tal associação seria a tentativa da criança ou adolescente de organizar o sentido da experiência violenta, podendo ocorrer condutas prejudiciais ao desenvolvimento psíquico, gerando sentimentos negativos e comportamentos agressivos, que poderiam levar aos transtornos ansiosos, de conduta e afetivos.

O estudo de Malik ${ }^{77}$ realizado com 117 crianças examinou a relação entre exposição à violência e sintomas de internalização, como sintomas de ansiedade, depressão, retraimento, além das manifestações somáticas, e externalização, como agressividade, impulsividade e comportamento desafiador. O estudo demonstrou que a violência comunitária foi relacionada aos problemas de internalização, enquanto a exposição à violência doméstica foi relacionada apenas com problemas de externalização, afirmando ainda que os diferentes tipos de violência ameaça o senso de segurança da criança, prejudicando o seu crescimento e desenvolvimento. Já com adolescentes, um estudo seccional brasileiro investigou a presença de fatores de risco e protetores para problemas de saúde mental em adolescentes, dentre os quais a violência intrafamiliar e urbana. Esse estudo mostrou que adolescentes expostos a essas situações mostraram ter duas vezes mais problemas de internalização e externalização ${ }^{78}$. A combinação entre violência e problemas de internalização e externalização chama atenção para o fato de esses problemas possuírem maior probabilidade de evoluírem para quadros clínicos na adolescência e também na vida adulta ${ }^{79}$.

Outro fator ambiental associado à ocorrência de transtornos foi a configuração familiar. Viver em um ambiente com pais separados se mostrou como fator de risco para qualquer transtorno mental em pelo menos três estudos desta revisão. Ainda segundo Nock et al. ${ }^{12}$, ser adotado aumentou em três vezes a chance de suicídio entre adolescentes. Em estudo de revisão, $\mathrm{Fu}^{80}$ relata que a condição de viver em um lar substituto parece aumentar a possibilidade de sofrimento mental na infância. Segundo Hersov ${ }^{81}$, o fato de ser adotado pode ser um fator de risco para transtorno mental quando o meio adotivo é inadequado (estressante e violento). Porém, a adoção atualmente tem sido definida como um fator de proteção para reduzir a presença de patologias psiquiátricas transmitidas geneticamente e que podem sofrer influência do ambiente externo ${ }^{81}$.

Ainda de acordo com o estudo de Roberts et al. ${ }^{33}$, viver com ambos os pais é um fator protetivo contra a ocorrência de qualquer transtorno mental. Do mesmo modo, Baptista et al. ${ }^{82}$ afirmam que a estrutura e o apoio familiar atenuam os efeitos dos eventos cotidianos estressantes, ou seja, pode prevenir o surgimento de algum distúrbio psicológico/psiquiátrico. Segundo revisão proposta por Souza et al. ${ }^{83}$, problemas nas relações familiares vêm sendo pesquisados como fatores dificultadores no tratamento dos transtornos mentais e agravamento desses em crianças e adolescentes. 


\section{CONCLUSÕES}

Os transtornos mentais mais prevalentes entre crianças e adolescentes foram depressão, transtornos de ansiedade, TDAH, por uso de substâncias e transtorno de conduta, associados principalmente com fatores biológicos, genéticos e ambientais, tais como sexo masculino, histórico familiar de transtorno mental, violência familiar e comunitária e configuração familiar. Poucos estudos de base populacional sobre transtornos mentais e fatores associados em crianças e adolescentes foram identificados. Estudos epidemiológicos de base populacional são importantes para que se conheçam a distribuição da exposição e do adoecimento e também as condições que influenciam a dinâmica dos padrões de risco em uma determinada comunidade.

Verifica-se uma carência na atenção à saúde mental infantojuvenil, não só em países em desenvolvimento, mas também em países desenvolvidos. Assim, identificar os transtornos mais prevalentes e seus fatores associados pode colaborar com a melhora na atenção e aumento da oferta de serviços específicos para população infantojuvenil ${ }^{5}$.

\section{CONTRIBUIÇÕES INDIVIDUAIS}

Daianna Lima Thiengo - Participou da busca bibliográfica e construção do artigo.

Maria Tavares Cavalcante - Participou da revisão do artigo.

Giovanni Marcos Lovisi - Participou da busca bibliográfica, construção e revisão do artigo.

\section{CONFLITOS DE INTERESSES}

Nada a declarar.

\section{AGRADECIMENTOS}

À Professora Doutora Lúcia Abelha, por suas contribuições neste artigo, à Coordenação de Aperfeiçoamento de Pessoal de Nível Superior (Capes) e ao Conselho Nacional de Desenvolvimento Científico e Tecnológico (CNPq).

\section{REFERÊNCIAS}

1. World Health Organization. Mental Health: New Understanding, New Hope. Genebra, World Health Organization; 2001

2. Organização das Nações Unidas. Objetivos de Desenvolvimento do Milênio: Mapa do Progresso de 2012. Nova York: Divisão de Estatística do Departamento de Assuntos Econômicos e Sociais; 2012.
3. Roberts ER, Attinksson CC, Rosenblatt A. Prevalence of psychopathology among children and adolescents. Am J Psychiatry. 1998;155(6):15-725.

4. Paula CS, Duarte CS, Bordin IA. Prevalence of mental health problems in children and adolescents from the outskirts of Sao Paulo City: treatment needs and service capacity evaluation. Rev Bras Psiquiatria. 2007;29(1):11-7.

5. Patel V, Flisher AJ, Hetricks S, McGorry P. Mental health of young people: a global publichealth challenge. Lancet. 2007;369(9569):1302-13.

6. Bordin IAS, Paula C. Estudos populacionais sobre saúde mental de crianças e adolescentes brasileiros. In: Mello MF, Mello AAF, Korh R. (Org.). Epidemiologia da saúde mental no Brasil. Porto Alegre: Artmed; 2007.

7. United States Department of Health and Human Services. Mental Health: A report of the surgeon general. In: Children and mental health. 1999. Disponível em: <http://www. surgeongeneral.gov/library/mentalhealth/toc.html\#chapter3>. Acesso em: 5 maio, 2014.

8. World Health Organization. Who Mental Policy and Service Guidance Package: Human Resources and Training in Mental Health. Genebra, World Health Organization; 2005.

9. Downs SH, Black N. The feasibility of creating a checklist for the assessment of the methodological quality both of randomised and non-randomised studies of health care interventions. J Epidemiol Community Health. 1998;52(6):377-84.

10. Silva TFC, Lovisi GM, Verdolin LD, Cavalcanti MT. Adesão ao tratamento medicamentoso em pacientes do espectro esquizofrênico: uma revisão sistemática da literatura. J Bras Psiquiatria. 2012;61(4):242-51.

11. Thiengo DL, Santos JFC, Mason VC, Abelha L, Lovisi GM. Associação entre apoio social e depressão durante a gestação: uma revisão sistemática. Cad Saúde Coletiva. 2011;19(2):12938.

12. Nock MP, Green JG, Hwang I. Prevalence, correlates, and treatment of lifetime suicidal behavior among adolescents results from the national comorbidity survey replication adolescent supplement. JAMA Psychiatry. 2013;70(3):300-10.

13. Lehaman S, Havik OE, Havil T, Heiervang E. Mental disorders in foster children: a study of prevalence, comorbidity and risk factors. Child Adolesc Psychiatry Ment Health. 2013;7(1):39.

14. Kinyanda E, Kizza R, Abbo C, Ndyanabangi S, Levin J. Prevalence and risk factors of depression in childhood and adolescence as seen in four districts of North-Eastern Uganda. BMC Int Health Hum Rights. 2013;13:19.

15. Madruga CS, Laranjeira R, Caetano R, Pinsky I, Zaleski M, Ferri CP. Use of licit and illicit substances among adolescents in Brazil--a national survey. Addict Behavior. 2012;37(10):1171-5.

16. Lewis KJS, Mars B, Lewis G, Rice F, Sellers R, Thapar AK, et al. Do parents know best? Parent-reported vs. child-reported depression symptoms as predictors of future child mood disorder in a high-risk sample. J Affect Disord. 2012;141(2-3):233-6.

17. Benjet C, Hernandéz-Montoya D, Borges G, Méndez E, Medina-Mora ME, Agular-Gaxiola S. Youth who neither study nor work: mental health, education and employment. Salud Publica Mex. 2012;54(4):410-7.

18. Vicente B, Saldivia S, La Barra F, Melipillan R, Valdivia M, Kohn R. Salud mental infantojuvenil en Chile y brechas de atención sanitarias. Rev Med Chile. 2012;140(4):447-57.

19. Ma X, Liu X, Hu X, Qiu C, Wang Y, Huang Y, et al. Risk indicators for post-traumatic stress disorder in adolescents exposed to the 5.12 Wenchuan earthquake in China. Psychiatry Res. 2011;189(3):385-91.

20. Siddique S, Banerjee M, Ray MR, Lahiri T. Attention-deficit hyperactivity disorder in children chronically exposed to high level of vehicular pollution. Eur J Pediatric. 2011;170(7):923-9.

21. Peng M, Liu A, Zhou J, Wen S, Li S, Yang T, et al. Association between posttraumatic stress disorder and preflood behavioral characteristics among children aged 7-15 years in Hunan, China. Med Princ Pract. 2010;20(4):336-40.

22. Vicente B, Saldivia S, Rioseco P, La Barra F, Valdivia M, Melipillan R,et al. Epidemiología de trastornos mentales infanto-juveniles en la Provincia de Cautín. Rev Med Chile. 2010;138(8):965-73.

23. Merikangas KR, He JP, Burstein M, Swanson SA, Avenevolis S, Cui L, et al. Lifetime prevalence of mental disorders in U.S. adolescents: results from the national comorbidity survey replication-adolescent supplement (NCS-A). J Am Acad Child Adolesc Psychiatry. 2010;49(10):980-9. 
24. Johnson S, Hollis C, Kochhar P, Hennessy E, Wolke D, Marlow N. Psychiatric disorders in extremely preterm children: Iongitudinal finding at age 11 years in the EPICure Study. J Am Acad Child Adolesc Psychiatry. 2010;49(5):453-63.

25. Zinzow HM, Ruggiero KJ, Resnick H, Hanson R, Smith D, Sauders B, et al. Prevalence and mental health correlates of witnessed parental and community violence in a national sample of adolescents. J Child Psychol Psychiatry. 2008:50(4):441-50.

26. Allen KL, Byrne SM, Forbes D, Oddy WH. Risk factors for full- and partial-syndrome early adolescent eating disorders: a population-based pregnancy cohort study. I Am Acad Child Adolesc Psychiatry. 2009;48(8):800-9.

27. Ouyang L, Fang X, Mercy J, Perou R, Grosse SD. Attention-deficit/hyperactivity disorder symptoms and child maltreatment: a population-based study. J Pediatric. 2008;153(6):851-6

28. Cruzeiro ALC, Silva RA, Horta BL, Souza LDM, Faria AG, Pinheiro RT, et al. Prevalência e fatores associados ao transtorno da conduta entre adolescentes: um estudo de base populacional. Cad Saúde Pública. 2008;24(9):2013-20.

29. Shanahan L, Copeland W, Costello EJ, Angold A. Specificity of putative psychosocial risk factors for psychiatric disorders in children and adolescents. J Child Psychol Psychiatry. 2008;49(1):34-42.

30. Bohon C, Stice E, Burton E, Fudell M, Nolen-Hoeksema S. A prospective test of cognitive vulnerability models of depression with adolescent girls. Behav Ther. 2008;39(1):79-90.

31. Piyavhatkul N, Pairojkul S, Suphakunpinyo C. psychiatric disorders in tsunami-affected children in Ranong Province, Thailand. Med Princ Pract. 2008;17(4):290-5.

32. Costello EJ, Worthaman C, Erkamili A, Angold A. Prediction from low birth weight to female adolescent depression: a test of competing hypotheses. Arch Gen Psychiatry. 2007;64(3):338-44.

33. Roberts RE, Roberts CR, Xing Y. Rates of DSM-IV psychiatric disorders among adolescents in a large metropolitan area. J Psychiatr Res. 2007;41(11):959-67.

34. Latimer W, Floyd LJ, Kariiis T, Novotna G, Exnerova P, O'Brien M. Peer and sibling substance use: predictors of substance use among adolescents in Mexico. Rev Panam Salud Pública. 2004;15(4):225-32

35. Canino G, Shrout PE, Rubio-Stipec M, Bird HR, Ramirez R, Chavez L, et al. The DSM-IV rates of child and adolescent disorders in Puerto Rico - Prevalence, correlates, service use, and the effects of impairment. Arch Gen Psychiatry. 2004;61(1):85-93.

36. Glowinski AL, Madden PA, Bucholz KK, Lynskey MT, Heath AC. Genetic epidemiology of self-reported lifetime DSM-IV major depressive disorder in apopulation-based twin sample of female adolescents. J Child Psychol Psychiatry. 2003;44(7):988-96.

37. Mártinez-González MA, Gual P, Lahortiga F, Alonso Y, Irala-Estévez J, Cervera S. Parental factors, mass media influences, and the onset of eating disorders in a prospective population-based cohort. Pediatrics. 2003;111(2):315-20.

38. Foley DL, Pickles A, Simonoff E, Maes HH, Silberg JL, Hewit JK, et al. Parental concordance and comorbidity for psychiatric disorder and associate risks for current psychiatric symptoms and disorders in a community sample of juvenile twins. J Child Psychol Psychiatry. 2001;42(3):381-94

39. Quintana MI, Gastal FL, Jorge MR, Miranda CT, Andreoli SB. Validity and limitations of the Brazilian version of the Composite International Diagnostic Interview (CIDI2.1). Rev Bras Psiquiatria. 2007;29(1):18-22.

40. Krueger RF, Watson D, Barlow DH. Toward a dimensionally based taxonomy of psychopathology. J Abnorm Psychol. 2005;114(4):491-3.

41. Bahls SC, Bahls FR. Psicoterapias da depressão na infância e na adolescência. Estud Psicol. 2003;20(2):25-34.

42. Goldberg AE. (How) does it make a difference? Perspectives of adults with lesbian, gay, and bisexual parents. Am J of Orthopsychiatry. 2007;77:550-62.

43. Mehler-Wex C, Kolch M. Depression in children and adolescents. Deutch Arztebl Int. 2008;105(9):149-55

44. Wright-Strawderman C, Watson BL. The prevalence of depressive symptoms in children with learning disabilities. J Learn Disabil. 1992;25(4):258-64.

45. Bernstein GA, Borchadt CM, Perwien AR. Anxiety disorders in children and adolescents: a review of the past 10 years. J Am Acad Child Adolesc Psychiatry. 1996;35(9):1110-9.

46. Golfeto JH, Barbosa GA. Epidemiologia. In: Rohde LA, Matos P. organizadores. Princípios e práticas em TDAH. Porto Alegre: Artmed, 2003.
47. Reiff MI, Stein MT. Attention-deficit/hyperactivity disorder evaluation and diagnosis: a practical approach in office practice. Pediatr Clin N Am. 2003;50(5):1019-48.

48. Vasconcelos MM, Werner JJ, Malheiros AFA, Lima DFN, Santos I, Barbosa JB. Prevalência do transtorno de déficit de atenção/hiperatividade numa escola pública primária. Arq Neuropsiquiatria. 2003;61(1):67-73.

49. Rohde LA, Filho ECM, Benetti L, Gallois C, Kieling C. Transtorno de déficit de atenção/ hiperatividade na infância e na adolescência: considerações clínicas e terapêuticas. Rev Psiq Clín. 2004;31(3):124-31.

50. Biederman J, Mick E, Faraone SV. Age-dependent decline of symptoms of attention deficit hyperactivity disorder: impact of remission definition and symptom type. Am J Psychiatry. 2000;157(5):816-8

51. Monitoring The Future Study. National Survey Results on drug use 1991 - 1997. Disponível em: www.isr.umich-edu:src:mtf. Acesso em: 28 maio, 2014.

52. Watkins TR, Lewellen A, Barret M. Dual diagnosis: an integrated approach to treatment. Sage publications, Inc; 2001.

53. Batistoni SS, Neri A L, Cupertino AP. Validity of the Center for Epidemiological Studies Depression Scale among Brazilian elderly. Rev Saúde Pública. 2007;41(4):598-605.

54. Galduróz JCF, Noto AR, Carlini EA. Tendências do uso de drogas no Brasil: síntese dos resultados obtidos sobre 0 uso de drogas entre estudantes de $1^{\circ}$ e $2^{\circ}$ graus em 10 capitais brasileiras. São Paulo: Centro Brasileiro de Informaç̧ões Sobre Drogas Psicotrópicas. CEBRID/UNIFESP; 2005.

55. Hinshay SP, Andersen CA. Conduct and oppositional defiant disorders. In: EJ Mash, RA Barkley. Child psychopathology, 2.ed. New York: The Guillford Press; 1996.

56. Offord DR, Boyle MH, Racine Y. Ontario Child Health Study: correlates of disorder. J Am Acad Child Adolesc Psychiatry. 1989;28(6):856-60.

57. Perales A, Soci C. Perspectiva sociocultural de las conductas disociales em adolescentes. Acta Psiquiatr Psicol Am Lat. 1998;44(1):29-39.

58. Vloet TD, Herpetz S, Herpetz-Dahlamann B. A etiology and life-course of conduct disorder in childhood: risk factors for the development of an antisocial personality disorders. Z Kinder Jugend Psychiatr Psychother. 2003;34:101-14.

59. Bordin IAS, Offord DR. Transtorno da conduta e comportamento anti-social. Rev Bras Psiquiatria. 2000;22(Suppl II):12-5.

60. Gadia CA, Tuchman R, Rotta NT. Autismo e doenças invasivas de desenvolvimento. J Pediatr. 2004;80(Supl II):s83-s94.

61. Croen LA, Grether JK, Selvin $S$. The epidemiology of mental retardation of unknown cause. Pediatrics. 2001;107(6):E86.

62. Borba LO, Schawarts E, Kantorski LP. A sobrecarga da família que convive com a realidade do transtorno mental. Acta Paul Enferm. 2008;28(4):588-94.

63. Campos PHF, Soares CB. Representação da sobrecarga familiar e adesão aos serviços alternativos em saúde mental. Psicol Rev. 2005;11(18):219-37.

64. Bierderman J, Faraone SV, Mick E. Clinical correlates of ADHD in females: findings from a large group of girls ascertained from pediatric andpsychiatric referral sources. J Am Acad Child Adolesc Psychiatry. 1999;38(8):966-7.

65. Guimarães NM, Pasian SR. Agressividade na adolescência: experiência e expressão da raiva. Psicol Estud. 2006;11(1):89-97.

66. Moon DG, Hecht ML, Jackson KM, Spellers RE. Ethnic and gender differences and similarities in adolescent drug use and refusals of drug offers. Subst. Use Misuse. 1999;34(8):1059-83.

67. Wood JT. Gendered lives: Communication, Gender, and Culture. Belmont, CA, Wadsworth Publishing; 1997

68. Costa IER, Ludemir AB, Avelar I. Violências contra adolescentes: diferenciais segundo estratos de condição de vida e sexo. Ciênc Saúde Coletiv. 2007;12(50):1193-200.

69. Oshiro FAB. Depressão e violência familiar: estudo de irmãos vítimas de maus-tratos e abandono [dissertação]. Campinas (SP): Instituto de Psicologia, Pontifícia Universidade Católica de Campinas; 1994.

70. Versiani M, Reis R, Figueira I. Diagnóstico do transtorno depressivo na infância e adolescência. J Bras Psiquiatria. 2000;49(10-12):367-82.

71. Andrade LHSG, Viana MC, Silveira CM. Epidemiologia dos transtornos psiquiátricos na mulher. Rev Psiq Clínica. 2006;33(2):43-54. 
72. Zhang B, Wing YK. Sex differences in insomnia: a meta-analysis. Sleep. 2006;29(1):85-93.

73. Santos M, Richards CS, Bleckley MK. Comorbidity between depression and disordered eating in adolescents. Eating Behaviors. 2007;8(4):440-9.

74. McVey G, Tweed S, Blackmore E. Healthy schools-healthy kids: a controlled evaluation of a comprehensive universal eating disorder prevention program. Body Image. 2007;4(2):115-36.

75. Michelon $L$, Vallada H. Fatores genéticos e ambientais na manifestação do transtorno bipolar. Rev Psiq Clínica. 2005;32(supl I):21-7.

76. Flores RZ, Carminha RM. Violência sexual contra crianças e adolescentes: algumas sugestões para facilitar o diagnóstico correto. Rev Psiquiatr Rio Gd Sul. 1994;16(2):158-67.

77. Malik NM. Exposure to domestic and community violence in a nonrisk sample. J Interpersonal Violence. 2008;23(4):490-504.
78. Paula CS, Vedovato MS, Bordin IAS, Barros MGSM, D'Antino MEF, Mercadante MT. Mental health and violence among sixth grade students from a city in the state of São Paulo. Rev Saúde Pública. 2008:42(3):524-8.

79. Pacheco J, Alvarenga P, Reppold C, Piccinini CA, Hutz CS. Estabilidade do comportamento anti-social na transição da infância para a adolescência: uma perspectiva desenvolvimentista. Psicol Reflex Crit. 2005;18(1):55-61.

80. Fu lL. Adoçäo e saúde mental. Infanto Rev Neuropsiquiatria. 2000;8(2):81-9.

81. Hersov L. Adoption. In: Rutter M, Taylor E, Hersov L. editors. 3.ed. Child and adolescent psychiatry: modern approaches. Oxford: Black-well Scientific, 1994.

82. Baptista MN, Baptista ASD, Dias RR. Estrutura e suporte familiar como fatores de risco na depressão de adolescentes. Psicol Cienc Prof. 2001;21(2):52-61.

83. Souza J, Abade F, Migliorini P, Silva C, Furtado EF. Avaliação do funcionamento familiar no contexto da saúde mental. Rev Psiq Clínica. 2011;38(6):254-9. 